\title{
Profile of pembrolizumab in the treatment of head and neck squamous cell carcinoma: design development and place in therapy
}

This article was published in the following Dove Press journal:

Drug Design, Development and Therapy

31 August 2017

Number of times this article has been viewed

\author{
Sulsal Haque ${ }^{1,2}$ \\ Mahender Yellu ${ }^{1,2}$ \\ Jaskirat Randhawa ${ }^{1,2}$ \\ Nooshin Hashemi-Sadraei $i^{1,2}$ \\ 'Division of Hematology/Oncology, \\ Department of Medicine, University \\ of Cincinnati, ${ }^{2}$ University of Cincinnati \\ Cancer Institute, Cincinnati, OH, USA
}

Correspondence: Nooshin Hashemi-Sadraei

Division of Hematology/Oncology, Department of Medicine, University of Cincinnati, Vontz Center for Molecular Studies, ML 0562, 3125 Eden Avenue, Cincinnati, OH 45267, USA

$\mathrm{Tel}+\mathrm{I} 5135582158$

Fax + I 5 I3 $5582 \mid 24$

Email hashemnn@ucmail.uc.edu
Abstract: Head and neck squamous cell cancer (HNSCC) is the sixth most common malignancy worldwide, and despite advances in cytotoxic, surgical and radiation techniques, outcomes are still poor in those with both locally advanced and metastatic diseases. The need for development of better therapeutics along with a greater understanding of the relationship between the immune system and malignancies has led to a new therapeutic modality, immune modulators, particularly checkpoint inhibitors in HNSCC. It is now well recognized that HNSCC circumvents crucial pathways utilized by the immune system to escape surveillance. These hijacked pathways include impairing tumor antigen presentation machinery and co-opting checkpoint receptors. This understanding has led to the development of monoclonal antibodies targeting checkpoint receptors and has resulted in promising outcomes in HNSCC. This article describes the mechanisms that HNSCC utilizes to escape immune surveillance, clinical impact of checkpoint inhibitors (with a focus on pembrolizumab), ongoing studies, and future directions.

Keywords: pembrolizumab, head and neck cancer, MK-3475, immunotherapy

\section{Design Introduction}

Head and neck squamous cell carcinoma (HNSCC) is the sixth most common cancer type worldwide and accounts of $\sim 350,000$ deaths per year. ${ }^{1,2}$ Risk factors such as tobacco, alcohol use, and more recently human papillomavirus (HPV) have been identified as etiologies for the development of HNSCC. Despite advances in the treatment of localized HNSCC, $15 \%-50 \%$ of patients will develop recurrent disease ${ }^{3}$ and these recurrences often account for the majority of deaths in these patients. In addition, increase in HPV-related malignancies, along with improved loco-regional control in many cases, has resulted in a surge of patients with distant metastatic disease, which was relatively uncommon decades ago.

In the recurrent/metastatic setting, historically platinum-based cytotoxic chemotherapy, with or without epidermal growth factor receptor (EGFR) inhibition, remains standard of care in the first-line setting. Despite high rates of toxicity $(70 \%-80 \%$ grade 3-4 adverse events [AEs]), response rates are in the range of $20 \%-36 \%$ and median survival is between 6 and 10 months. ${ }^{4}$ Among platinum ineligible patients, or in those with progression after platinum therapy, single-agent cytotoxics or singleagent EGFR therapy, including EGFR tyrosine kinase-inhibiting drugs and monoclonal antibodies targeting the receptor, has been extensively studied with minimal responses and no significant survival advantage. ${ }^{5,6}$ In many instances, patients have previously 
been exposed to bi- or tri-modality treatment involving surgery, radiation, and chemotherapy and, due to their limited performance statuses and comorbidities, are often unable to tolerate such aggressive treatment regimens. Hence, there is an unmet need for newer treatments to improve outcomes with reasonable toxicity profiles. We discuss the rationale for immune-modulating therapies in HNSCC with an emphasis on pembrolizumab, clinical data, and future directions.

\section{The immune system's relationship for the development of HNSCC}

Tumors produce proteins that are not normally evident in healthy cells, and this can occur as a result of altered DNA repair mechanisms, ${ }^{7}$ somatic mutations, ${ }^{8}$ or viruses $;{ }^{9}$ these proteins are known as tumor-associated antigens (TAAs). These abnormal proteins are recognized as antigens by the immune system and can activate the immune system. The immune system in turn utilizes both its innate and adaptive components to confer protection from tumors by recognizing self from altered-self. The innate immune system is activated first and directs NK cells and macrophages toward tumor cells followed by the adaptive immune system, which mediates a more tumor-specific response.

The adaptive immune response is reliant on lymphocytes, which include CD8 cytotoxic T lymphocytes (CTL), CD4 helper $\mathrm{T}$ cells, regulatory $\mathrm{T}$ cells (Tregs), and B cells. The $\mathrm{T}$-cell response leads to direct cell death by utilizing cytokines, perforin, and granzyme, while B cells secrete antibodies and use antibody-dependent cellular cytotoxicity for tumor cell death.

T-cell responses are triggered by TAAs and presented to antigen-presenting cells (APCs) such as dendritic cells with the aid of toll-like receptor (TLR) ligands. The presence of TAAs on APCs initiates an effector T-cell response. This signal is insufficient on its own and requires further co-stimulation by B7:CD28 receptor complex. Interleukin-12 (IL-12) and type I interferon (INF) are also activated to avoid T-cell tolerance. ${ }^{10}$ Regulation of $\mathrm{T}$-cell responses can be augmented further by OX40 or 4-1BB or downregulated by programmed death-1 (PD-1) or cytotoxic T-lymphocyte antigen-4 (CTLA-4). ${ }^{11}$ Once the effector $\mathrm{T}$ cells are fully activated, they can be directed toward tumor cells leading to cell death.

\section{Immune escape and immune suppression in HNSCC}

Immune surveillance, which was first described years ago, identified that premalignant cells can be recognized and removed by the immune system through the mechanisms described earlier. ${ }^{12}$ The important relationship between an intact immune system and oncogenesis is evident by the increased incidence of malignancies in immunocompromised patients. ${ }^{13,14}$ For example, HNSCC has been reported to occur more frequently in those who have a renal or bone marrow transplant as well as HIV patients. ${ }^{13,14}$ The vast majority of patients who develop HNSCC are immunocompetent, yet tumors have been able to proliferate and metastasize by creating mechanisms to evade the immune system.

An effective immune response hinges on a system of signals that includes TAAs being presented to APCs, activation of co-stimulatory and co-inhibitory signals, and immune activating cytokines. This system can get hijacked in solid tumors, including HNSCC resulting in ineffective TAA presentation, impaired tumor cell eradication, and creation of an unwelcoming tumor microenvironment (TME). This makes it challenging for immune cells, including $\mathrm{T}$ cells to remain active and penetrate tumor tissue effectively, all of which contribute to tumor growth and metastasis.

Human leukocyte antigen (HLA) plays a critical role in activating both $\mathrm{T}$ cells and NK cells, which in turn results in tumor cell eradication. ${ }^{15}$ Altering this interaction is an important immune escape mechanism for malignancies. ${ }^{16,17}$ Abnormal expression and function of HLA machinery leads to impaired antigen processing and presentation and has been reported in up to $20 \%$ of HNSCC patients correlating with lymph node metastasis and poor prognosis. ${ }^{18,19}$ Furthermore, EGFR expression, which occurs in $80 \%$ of HNSCC, may also play a role in reducing HLA expression through mitogenactivated protein kinase (MAPK) and phosphatidyl inositol 3-kinase/protein kinase B (PI3K/AKT) pathways. ${ }^{20}$

The TME in HNSCC is integral in escaping immune surveillance and promoting an immunosuppressive environment as it contains a large number of Tregs and myeloidderived suppressor cells (MDSCs). Tregs are derived from improperly activated $\mathrm{T}$ cells, and MDSCs originate from myeloid cells. ${ }^{21}$ Tregs produce immune checkpoint receptors including CTLA-4, TIM-3, and PD-L1 and express transforming growth factor-beta (TGF- $\beta$ ), all of which contribute to T-cell tolerance and exhaustion. ${ }^{22}$ MDSCs secrete inflammatory mediators including IL-1, IL-6, reactive oxygen species, and inducible nitric oxide synthase suppressing APC mechanisms. ${ }^{21}$ In HNSCC MDSCs can deactivate T-cell responses by upregulating PD-L1 expression, releasing TGF- $\beta$, as well as increasing arginase- 1 activity through STAT3 production. . $^{23,24}$

In addition to immune escape mechanisms, HNSCC is an immunosuppressive disease. HNSCC patients have 
lower absolute lymphocyte counts, ${ }^{25}$ impaired NK cell production, ${ }^{26}$ and can also produce TGF- $\beta$, IL-10, or vascular endothelial growth factor (VEGF) that further contributes to impaired immune responses. ${ }^{27,28}$ Altered penetration of tumor-infiltrating $\mathrm{T}$ lymphocytes has also been reported in HNSCC and other malignancies. ${ }^{29}$

\section{Immune checkpoint pathways in HNSCC}

Checkpoint receptors are the immune system's counter mechanism to thwart excessive immune system activation and prevent autoimmune diseases, and this process is known as adaptive immune system resistance. PD-1 and CTLA-4 are two of the most well studied coinhibitory checkpoint receptors, and their overexpression in malignancies has been recognized as a key pathway in escaping immune surveillance. Other costimulatory and coinhibitory checkpoint signals have been identified as well. LAG3, TIM3, and killer immunoglobulin-like receptor (KIR) are coinhibitory signals and when expressed lead to T-cell apoptosis, preventing appropriate T-cell-directed cell death. ${ }^{11,30}$ Costimulatory signals including CD137, OX40, and CD40L are important in directing appropriate $\mathrm{T}$-cell responses, and their decreased expression on T cells has been reported in HNSCC specimens and appears to have a correlation with worse outcomes. ${ }^{11,31}$

\section{CTLA-4 checkpoint receptor}

CTLA-4 receptor is primarily found in Tlymphocytes and binds to $\mathrm{B} 7$ in APCs in response to T-cell receptor activation. ${ }^{32,33}$ While CTLA-4 does engage with $\mathrm{CD}^{+}$cytotoxic T cells, it does so in a transient manner ${ }^{34}$ whereas it is highly expressed on $\mathrm{CD}^{+}{ }^{+}$Tregs, and this is the driving mechanism in dampening down T-cell-mediated immune responses. ${ }^{35,36}$ Furthermore, CTLA-4 expression can lead to B7 ligand degradation via endocytosis further hampering the activation of $\mathrm{T}$ cells. ${ }^{37,38}$ Preclinical work has revealed that blocking CTLA-4 activity in tumors led to delayed tumor growth and restoration of antitumor immunity. ${ }^{39}$ This has resulted in the development of two monoclonal antibodies directed against CTLA-4, ipilimumab, and tremelimumab. Ipilimumab has been approved by the US Food and Drug Association (FDA) as a single agent in melanoma, ${ }^{40}$ and there are currently a number of trials underway utilizing these drugs in combination with other immune-modulating drugs or cytotoxic therapies. ${ }^{41}$

\section{PD-I checkpoint receptor}

PD-1 is typically upregulated in activated lymphocytes including T cells, B cells, monocytes, and NK cells and binds to PD-L1 and PD-L2 ligands. ${ }^{42}$ Many cells broadly express
PD-L1 including hematopoietic cells, pancreatic islet cells, endothelial cells, and epithelial cells, and this increased expression confers protection from immune-mediated injury. ${ }^{43,44}$ PD-L2 expression, however, is restricted to APCs and can effectively curb activated T-cell responses in the setting of inflammation. ${ }^{45}$ PD-L1 and PD-L2 expressions are initiated by inflammatory cytokines including INF- $\gamma$, tumor necrosis factor-alpha (TNF- $\alpha$ ), IL-4, and granulocyte macrophage colony-stimulating factor (GM-CSF). ${ }^{44,46}$ Once PD-1 binds to PD-L1 or PD-L2, the immune system activation is effectively turned off by decreasing Th1 cytokines' secretion, inhibiting $\mathrm{T}$-cell proliferation and preventing CTL-induced cell death. ${ }^{47,48}$

This pathway, however, is co-opted in pathological states such as infections and malignancies. For example, the TME employs proinflammatory cytokines such as INF- $\gamma$ to induce PD-L1 expression. ${ }^{49}$ In addition, tumor cells have developed intrinsic mechanisms such as PI3K-AKT and JAK/STAT pathways to upregulate PD-L1 expression on their cell surfaces. ${ }^{50,51}$ As a result of PD-L1 overexpression, tumorspecific $\mathrm{T}$ cells cannot be activated to mount an effective response and, therefore, enter the state of T-cell exhaustion.

Degree of expression of PD-L1 appears to correlate with tumor grade in various tumors including gastric, ovarian, lung, and renal cancers and can be expressed on both primary and metastatic sites. ${ }^{52-55}$ Approximately two-thirds of patients with HNSCC have some degree of PD-L1 expression. ${ }^{56,57}$ Expression of PD-L1 in HNSCC is induced by NK cells through INF- $\gamma$ secretion $^{50}$ but can also be increased by both chemotherapy and radiation therapy with tumor cells displaying upregulation of PD-1 for up to 1 year after treatment. ${ }^{57-59}$ Since many tumors make up the spectrum of HNSCC, the prognostic significance of PD-L1 within anatomical subsites, as well as its significance in the context of HPV status remains unclear. In addition, the wide-ranging cutoff points used in assessing PD-L1 expression and subjective scoring of PD-L1 on tumor cells further complicate its prognostic value.

Antibody blockade of this critical pathway has now emerged as a novel and effective therapeutic strategy in malignancies, and two PD-1 inhibitors, pembrolizumab and nivolumab, are FDA approved in a number of malignancies, including HNSCC.

\section{Development} Drug structure, pharmacokinetics (PK), and dosing

Pembrolizumab is a humanized monoclonal IgG4 kappa isotype anti-PD-1 antibody that hinders binding of PD-1 with 
its ligand PD-L1, allowing re-initiation of tumor-specific $\mathrm{T}$-cell responses.

Based on analysis from the pooled data of the KEYNOTE001, -002, and -006 studies of patients with advanced melanoma, non-small-cell lung cancer (NSCLC), and other solid tumor types, the PK properties show that pembrolizumab has low clearance with a small volume of distribution and displays similar PK properties as other therapeutic antibodies. ${ }^{59}$ Subsequent pooled analyses of earlier studies have also identified that weight-based dosing of pembrolizumab or a fixed dose resulted in similar distribution, clearance, and PK properties. Similarly, dosing the drug every 14 or 21 days was considered safe and efficacious. The half-life elimination is estimated to be $\sim 23$ days with a clearance rate of $0.22 \mathrm{~L} /$ day, which is not affected by age or gender. Furthermore, fixed dose regimens every 21 days provide advantages in terms of safety, convenience, and adherence..$^{60}$

\section{Pembrolizumab landmark studies Melanoma}

Pembrolizumab (formerly MK-3475) was evaluated in a Phase I trial, and it was administered intravenously at a dose of either $10 \mathrm{mg} / \mathrm{kg}$ of body weight every 2 or 3 weeks or $2 \mathrm{mg} / \mathrm{kg}$ every 3 weeks in patients with advanced melanoma and included those who had received previous treatment with a CTLA-4 inhibitor ipilimumab and those who had no prior treatment. ${ }^{61}$ The analysis showed a $38 \%$ overall response rate (ORR) (95\% confidence interval [CI], 25-44), with the highest confirmed response rate observed in the cohort that received $10 \mathrm{mg} / \mathrm{kg}$ every 2 weeks (52\%; 95\% CI, 38-66). This dose, however, was also associated with higher drugrelated AEs. The rates of responses did not vary between those who received ipilimumab previously and those who did not and was 38\% (95\% CI, 23-55) and 37\% (95\% CI, 36-49), respectively. ${ }^{61}$

The pivotal KEYNOTE-006 study was a Phase III study that compared two doses of pembrolizumab $(10 \mathrm{mg} / \mathrm{kg}$ every 3 weeks and $10 \mathrm{mg} / \mathrm{kg}$ every 2 weeks) with four doses of ipilimumab (3 mg/kg). ${ }^{62}$ Both arms demonstrated statistically significant improvements in progression-free survival (PFS) at 6 months (47\% for the 3-week arm, 46\% for the 2 -week arm, and $26 \%$ for ipilimumab arm) with a hazard ratio (HR) of $0.58(P<0.001)$. The study also found that overall survival (OS) was improved in both pembrolizumab arms compared to the ipilimumab arm with HR 0.63 in the 2-week arm $(95 \%$ CI, $0.47-0.83 ; P<0.001)$ and 0.69 in the 3-week arm $(95 \%$ CI, $0.52-0.90 ; P=0.004) .{ }^{62}$ These results led to the approval of pembrolizumab in the treatment of melanoma.

\section{NSCLC}

A number of clinical trials have been conducted assessing the efficacy of pembrolizumab in NSCLC. The KEYNOTE001 trial was a large Phase I study with multiple expansion cohorts and included 495 patients with NSCLC. ${ }^{63}$ Patients were assigned to receive pembrolizumab at either 2 or $10 \mathrm{mg} / \mathrm{kg}$ every 3 weeks or $10 \mathrm{mg} / \mathrm{kg}$ every 2 weeks. The ORR was $19 \%$ (95\% CI, 14-22) in previously treated patients $(n=394)$ and 24.8\% (95\% CI, 16.7-34.3) in treatment-naive patients $(n=101)$. Patients' outcomes were stratified by PD-L1 expression, and the trial noted that those with PD-L1 expression $>50 \%$ had better PFS in both treatment-naive and platinum failure patients, whereas PFS and OS were shorter in patients with PD-L1 expression either $<1 \%$ or $1 \%-49 \%$ respectively. ${ }^{63}$ This was followed by KEYNOTE-010 Phase II/III study that compared two different doses of pembrolizumab (2 or $10 \mathrm{mg} / \mathrm{kg}$ every 3 weeks) with docetaxel in relapsed refractory NSCLC in patients who had $>1 \%$ PD-L1 expression. ${ }^{64}$ Both arms of pembrolizumab ( 2 and $10 \mathrm{mg} / \mathrm{kg}$ ) showed better OS compared to docetaxel (10.4 vs 12.7 vs 8.5 months). Similar to KEYNOTE-001, median OS and PFS were highest among patients with PD-L1 expression $>50 \% .{ }^{64} \mathrm{KEYNOTE-024}$ was a pivotal Phase III trial that compared pembrolizumab with a platinum doublet chemotherapy in first-line treatment for 305 treatment-naive NSCLC patients who had a PD-L1 expression of $>50 \% .{ }^{65}$ Patients were given a fixed dose of $200 \mathrm{mg}$ every 3 weeks and results showed that median PFS was 10.3 months (95\% CI, 6.7 to not reached) in the pembrolizumab group vs 6 months $(95 \%$ CI, 4.2-6.2) in the chemotherapy arm. Estimated OS at 6 months was $80 \%$ in the pembrolizumab group vs $72 \%$ in the chemotherapy arm with an HR of $0.60(95 \%$ CI, $0.41-0.89, P=0.005)$. There were also fewer treatmentrelated serious AEs compared to chemotherapy arm (26\% vs $53 \%$ ). Based on these trials, pembrolizumab was approved for patients beyond first-line platinum therapy (for those with a PD-L1 expression of $>1 \%$ ) and in the first-line setting (for those with a PD-L1 expression of $>50 \%$ ).

\section{Place in therapy in head and neck cancers}

Patients with relapsed or metastatic HNSCC have poor outcomes with a median OS of 6-10 months. Current frontline standard of care for metastatic HNSCC remains a combination of chemotherapy with a platinum backbone with or without cetuximab. However, duration of disease control is short and progression of after-platinum-based therapy is associated with dismal outcomes in a generally frail patient population with disease- and treatment-related comorbidities. 
Pembrolizumab along with nivolumab are recently approved checkpoint inhibitors that have demonstrated durable clinical efficacy and survival advantage in the absence of significant high-grade toxicities. Details of the trials are discussed below.

\section{Nivolumab trial in HNSCC}

Nivolumab another PD-1 inhibitor is the only checkpoint inhibitor to demonstrate improved outcomes in metastatic HNSCC. CheckMate-141 was a large Phase III trial comparing nivolumab with single-agent chemotherapy in patients with HNSCC (oral cavity, larynx, and pharynx) progressing within 6 months after platinum therapy. ${ }^{57}$ The trial demonstrated a significantly improved OS in patients treated with nivolumab (7.5 vs 5.5 months) with an HR of 0.70 , and 1-year survival rates were higher in the nivolumab group than in the chemotherapy group (36.6\% vs $16.6 \%$ ). There was no difference in PFS between the two arms, 2 months in the nivolumab group vs 2.3 months, but the study did report a late break in the survival curves. At the time of publication, $19.7 \%$ of patients in the nivolumab group had no disease progression at 6 months vs $9.9 \%$ of patients in the chemotherapy arm. The ORR was $13 \%$ including six complete responses vs $5.8 \%$ in the chemotherapy group, and the median time to response was 2 months in both arms. More importantly, responses were durable in the nivolumab group in comparison to the chemotherapy arm. Nivolumab also had lower AE rates; all grade $\mathrm{AE}$ rates were $59 \%$ in the nivolumab arm vs $77 \%$ in the chemotherapy arm, and grade 3 or 4 AEs were $13 \%$ in the nivolumab group vs $35 \%$ in the chemotherapy arm. These survival rates in combination with its tolerability were very encouraging, particularly considering the patient selection criteria (pretreated and progressed shortly after platinum) in comparison to historical studies. Based on these data, the FDA approved nivolumab in HNSCC progressing after platinum therapy.

\section{Pembrolizumab trials in cancers arising from the head and neck region}

KEYNOTE-012 was a multicenter open-label Phase Ib study that included 60 patients with HNSCC with a PDL-1 level of $\geq 1 \%$ on tumor cells or stroma by immunohistochemistry (IHC). ${ }^{59}$ This study, unlike CheckMate-141, did not mandate rapid progression within 6 months postplatinum exposure. Patients had a higher rate of HPV-positive disease (38\%) in comparison to CheckMate- $141,82 \%$ were male and $85 \%$ had received at least one prior therapy. All patients were treated with pembrolizumab $10 \mathrm{mg} / \mathrm{kg}$ every 2 weeks. Toxicity was manageable, with a grade 3 or $4 \mathrm{AE}$ rate of $17 \%$, which included an increase in aspartate aminotransferase and alanine aminotransferase, hyponatremia, and drug-related rash. Twenty-seven of the 60 patients (45\%) experienced a serious adverse effect, but no drug-related deaths were reported. The ORR was $18 \%$ with a $25 \%$ ORR in HPVpositive patients and a $14 \%$ ORR in HPV-negative patients. ${ }^{59}$ Similar to CheckMate-141, the median time to response was 2 months, the median duration of response was 53 weeks (13 to not reached), and eight of the 45 evaluable patients had ongoing responses at time of publication.

This was followed by an expansion cohort of the KEYNOTE-012 study and included 132 patients with relapsed/recurrent HNSCC who were given a fixed dose of pembrolizumab at $200 \mathrm{mg} .{ }^{60}$ The expansion cohort did not require patients to have a PD-L1 expression of at least $1 \%$. In this study, $83 \%$ of patients were males and $56 \%$ had at least $\geq 2$ lines of therapy. The trial demonstrated an objective response rate of $18.2 \%$ in this 132 patient cohort ( 99 evaluable) with 18 partial responses, and $>30 \%$ had stable disease. The median time to response was $2-11$ months, and median duration of response was not reached. The PFS and OS at 6 months were 23 and 59\%, respectively. Drug-related adverse effects were noted in $47 \%$ of patients, with grade $\geq 3$ in $9 \%$. Most common AEs of any grade were fatigue (21\%), hypothyroidism (11\%), loss of appetite (7\%), and nausea $(5 \%)$. When stratified by HPV status, there were higher response rates seen in the HPV-positive group vs HPVnegative group ( $32 \%$ vs $14 \%$, respectively). The median OS in this trial was 13 months, which was similar to the reported OS of 10.1 months in the EXTREME trial. ${ }^{4}$

Based on these results, FDA granted accelerated approval for the use of pembrolizumab in recurrent or metastatic HNSCC with disease progression on or after platinum-based therapy.

Biomarker evaluation of the expanded cohort of KEYNOTE-012 was also conducted. Interestingly, this trial showed that while PD-L1 on tumor cells did not predict response, PD-L1 expression of $\geq 1 \%$ on both tumor and inflammatory cells did predict an improved ORR. When both tumor and immune cells had a PD-L1 expression of $\geq 1 \%$, there was a $22 \%$ ORR vs $1 \%$ in those who were negative. ${ }^{60}$ Similarly, patients who had higher PD-L1 expression on both tumor and mononuclear inflammatory cells were noted to have a better PFS $(P=0.026)$ and $\mathrm{OS}(P=0.008)$, but again this significance was lost when restricted to only tumor cells. Exploratory analysis of PD-L2 and IFN-y signature was found to correlate with clinical response. ${ }^{59,60}$ 
Pembrolizumab's efficacy has also been tested in patients who have progressed on platinum and cetuximab therapies. KEYNOTE-055 evaluated pembrolizumab at a dose of $200 \mathrm{mg}$ every 3 weeks in 171 patients who were refractory to platinum and cetuximab therapies. PD-L1 expression was defined as $\geq 1 \%$ in both tumor and mononuclear inflammatory cells, but PD-L1 positivity was not needed to enter the study. ${ }^{66}$ The study reported an ORR of $16 \%$, and response rates were similar in both HPV-positive and HPV-negative diseases ( $16 \%$ and $15 \%$, respectively). The median time to response like other studies was 2 months, the median duration of response was 8 months ( $2+$ to $12+$ months), and 21 patients still had ongoing responses. PFS and OS was 2.1 and 8 months, respectively, and this did not differ by HPV status. Response rates were also stratified by both 1 and 50\% PD-L1 expressions. At PD-L1 expression $\geq 1 \%$, the ORR was $18 \%(12 \%-25 \%)$ vs $12 \%(2 \%-30 \%)$ in PD-L1-negative patients. Higher PD-L1 expression of $\geq 50 \%$ resulted in better responses vs those who had $<50 \%$ expression, $27 \%$ (95\% CI, $15 \%-42 \%$ ) vs $13 \%$ (95\% CI, 7\%-20\%), respectively ${ }^{66}$ It was also well tolerated in this heavily treated patient population with a grade $3-5 \mathrm{AE}$ rate of $16 \%$ including one death from pneumonitis.

\section{Pembrolizumab in nasopharyngeal carcinoma}

Nasopharyngeal carcinomas are either excluded (eg, EXTREME study $)^{4}$ or represent a minority of the population in head and neck trials (eg, only $5 \%$ of patients in KEYNOTE-012) and historically have a median OS of 11-18 months with platinum therapy ${ }^{67,68}$ Given the high percentage of virally driven cancers among nasopharyngeal carcinoma, different biologies compared to smoking-related HNSCC, and different patterns of progression (higher possibility of systemic metastasis in nasopharyngeal cancers), KEYNOTE-028 was conducted specifically to address this population in a multicohort Phase $\mathrm{Ib}$ trial. Twenty-seven patients with nasopharyngeal carcinoma were treated with pembrolizumab at $10 \mathrm{mg} / \mathrm{kg}$ every 2 weeks for 2 years. ${ }^{69}$ Seven patients experienced a partial response, and 14 patients had stable disease with an ORR of $25.9 \%$. Drug-related AEs of any grade occurred in $>70 \%$ of patients, and most common AEs were fatigue (37\%), pruritus (29.6\%), nausea (25.9\%), pyrexia (25.9\%), and myalgia (22.2\%). Grade $\geq 3$ AEs occurred in $\sim 30 \%$ patients. At the conclusion of the study, five patients were still receiving pembrolizumab treatment. ${ }^{69}$

\section{PD-LI expression in HNSCC}

Tumor infiltration of lymphocytes with $\mathrm{CD} 4^{+} / \mathrm{CD} 8^{+}$in patients with HNSCC is necessary for antitumor effect from checkpoint inhibitors and may also serve as a prognostic indicator. In one study, PD-L1 expression was described in 39 of the 45 patients with HNSCC and these patients with PD-L1 expression had a lesser degree of intratumoral infiltration of lymphocytes ( $P=0.047$ ) but expression did not appear to impact survival. ${ }^{70}$ It was also observed that degree of peritumoral $\left(\mathrm{CD} 8^{+}\right)$infiltration correlated with lymph node metastasis $(P<0.001)$, tumor size $(P=0.003)$, and clinical stage $(P<0.001) .^{70}$

In a larger retrospective study of $>300$ patients, PD-L1 expression in oral squamous cell carcinoma was analyzed for its association with clinical, demographic characteristics, and survival outcomes. ${ }^{71} \mathrm{PD}-\mathrm{L} 1$ expression was analyzed by two pathologists using IHC and was classified as high expression if staining was graded as 2-3. High PD-L1 expression did not have a significant relationship to tumor stage, nodal staging, or pathologic grade. However, female patients were more likely to have a higher PD-L1 expression compared to males $(P=0.0062)$ and those who developed distant metastases also had higher rates of high PD-L1 expression $(P=0.0103)$, which remained significant after multivariate analysis. ${ }^{71}$

In larger prospective studies of recurrent and metastatic HNSCC, PD-L1 expression is usually defined by $\geq 1 \%$ IHC expression in tumor and/or inflammatory cells within stroma based on archival tissue. Based on this definition, 57\%-78\% of patients are considered PD-L1 positive. ${ }^{57,59}$ In addition to variability in choice of antibodies and definition of positivity, studies are ongoing to address how age of archived tissue and exposure to certain therapies can modify PD-L1 IHC expression.

\section{Clinical and biomarker predictors of response and outcomes}

Recent data suggest that lower volume of disease and using pembrolizumab earlier in the course of treatment algorithms results in better responses. One large post hoc analysis of 200 patients demonstrated that patients who had received $\leq 2$ treatments had higher responses ( $32 \%$ vs $16 \%$ ) compared to those who received more treatments. ${ }^{72}$ This study also showed higher responses in patients with smaller volume of disease compared to those with larger volume of disease. ${ }^{72}$

At present, PD-L1 remains the only validated marker for predicting responses to checkpoint inhibitors with higher expression of PD-L1 correlating with better responses. This has been seen in larger trials for HNSCC as well as other malignancies. ${ }^{59,60}$ For example, in CheckMate-141, response rates were stratified by PD-L1 expression $\geq 1, \geq 5$, and $\geq 10 \%$ and were 17,22 , and $27.9 \%$, respectively. Patient responses based on PD-L1 expression $<10,<5$, and $<1 \%$ were 10 , 11 , and $12 \%$, respectively. PD-L1 expression $\geq 1 \%$ also had 
a better median OS than standard therapy, 8.7 vs 4.6 months (HR 0.55; 95\% CI, 0.36-0.83), whereas patients with PD-L1 expression $<1 \%$ had similar OS to standard therapy, 5.7 vs 5.8 months (HR 0.89; 95\% CI, 0.54-1.45). In KEYNOTE012 , patients enrolled into the trial required $\geq 1 \%$ PD-L1 expression in tumor cells, stroma cells, or inflammatory mononuclear cells and positive expression was associated with best overall response $(P=0.010)$ and PFS $(P=0.020)$. Similarly, in the expansion cohort of KEYNOTE-012 where PD-L1 expression was not needed for enrollment into trial, PD-L1 expression $\geq 1 \%$ was associated with a $22 \%$ ORR vs $1 \%$ in those who were negative. The KEYNOTE studies also highlighted that PD-L1 expression on both tumor cells and the TME may predict responses.

Despite the excitement around durable responses seen with pembrolizumab, most patients do not have a clinical benefit from pembrolizumab as response rates remain in the $10 \%-20 \%$ range and, therefore, a better understanding of identifying those who will truly respond to pembrolizumab is needed. A recent study has identified an inflamed phenotype defined by $>50 \% \mathrm{CD}^{+}$infiltration, $>50 \% \mathrm{PD}-1 / \mathrm{TIM} 3$ expression in the TME, as well as higher NK cell infiltration, ${ }^{73}$ and this inflamed phenotype correlated with better outcomes and was also more sensitive to PD-1 inhibitors in comparison to those who did not have this phenotype. ${ }^{73}$

An exploratory analysis on KEYNOTE-012 identified six INF- $\gamma$ regulated gene expressions among responders vs nonresponders to pembrolizumab. ${ }^{59}$ All responders in this trial had higher mean expressions of these genes, and this signature had a negative predictive value of $95 \%$, which may potentially identify groups of patients who will not benefit from pembrolizumab treatment. Other studies have suggested higher pretreatment $\mathrm{CD} 8^{+} \mathrm{T}$ cells, and T-cell receptor oligoclonality may be associated with better outcomes in melanoma patients. ${ }^{74}$

It is well known that HPV disease is associated with better outcomes $^{75}$ and that HPV-positive disease is biologically distinct from HPV-negative disease. ${ }^{76}$ Tumor specimens have also revealed that HPV-positive disease is associated with higher tumor inflammation and PD-L1 expression, ${ }^{77}$ but the complex interplay between PD-L1 expression, HPV status, and outcomes is still not clearly defined. The recent results of both the nivolumab and pembrolizumab trials have attempted to untangle this relationship. In the CheckMate-141 study, an exploratory analysis found that HPV-positive disease and/or PD-L1 expression 1\% resulted in a greater degree of benefit from nivolumab; however, these interactions were not significant. ${ }^{57}$ Furthermore, HPV-positive patients had a longer OS when treated with nivolumab compared to chemotherapy
(9.1 vs 4.4 months; HR 0.56 ; 95\% CI, 0.32-0.99). ${ }^{57}$ In the KEYNOTE-012 trial, the ORR was higher in patients with HPV-positive disease than with HPV-negative disease $(25 \%$ vs $14 \%$, respectively). ${ }^{59}$ Similarly, the KEYNOTE-12 expansion study revealed an ORR of $32 \%$ in HPV-positive disease compared to an ORR of $14 \%$ in HPV-negative disease. ${ }^{60}$ An improvement in PFS at 6 months was also seen in HPVpositive disease (37\% vs 20\%) and OS at 6 months also favored HPV-positive disease (70\% vs 56\%), although this was not statistically significant. ${ }^{60}$ It is important to note that while these trials suggest that patients with HPV-positive disease may demonstrate higher magnitude of clinical benefit from immune therapy, these were unplanned analyses and were not powered to detect the significance; hence, more data are needed to validate these outcomes.

It is also important to note that pseudoprogression (caused by T-cell infiltration and an apparent increase in tumor size), which was reported with CTLA-4 inhibitors in melanoma patients, ${ }^{78,79}$ is not a common occurrence in HNSCC. The KEYNOTE-012 only reported one patient who initially had radiographic progression followed by a reduction in tumor size among the 104 patients who were recruited to the trial ${ }^{59}$ suggesting that clinicians should exercise caution in patients who are noted to have increasing size in tumors after initiation of pembrolizumab or other PD-1 inhibitors, especially if this is accompanied with worsening clinical symptoms.

\section{Future directions}

The exciting development of PD-1 inhibitors in producing durable responses in HNSCC has led to new questions, namely how do we continue to improve magnitude and durability of clinical benefit and how to improve responses in those who are not high PD-L1 expressers? At the moment, pembrolizumab and other PD-1 inhibitors are being trialed with various other modalities including other immunomodulatory drugs, chemotherapy or radiation, or both.

\section{PD-I inhibitors with other immune- modulating drugs}

Based on the single-agent activity of pembrolizumab and other PD-1 inhibitors, there is a strong rationale for combining PD-1 inhibitors with CTLA-4 inhibitors. CTLA-4 is mostly involved in hampering early $\mathrm{T}$-cell activation within lymph nodes, and PD-1 is mostly responsible in the later stages of limiting T-cell function within tissue ${ }^{80}$ CTLA-4 inhibitors were the first checkpoint inhibitors to demonstrate clinical efficacy in large clinical trials. Preclinical work has shown that dual blockade led to more T-cell proliferation, increased production of cytokines, and better 
tumor regression. ${ }^{81}$ Importantly, this combination has already been tested in melanoma patients and has been approved by the FDA. While there are no large trials currently involving pembrolizumab and CTLA-4 inhibitors, there are several other late phase trials involving other PD-1/PD-L1 inhibitors in combination with CTLA-4 inhibitors. CONDOR (NCT02319044) is a randomized Phase II study evaluating this combination specifically in PD-L1-negative disease, and this is based on Phase I data in lung cancer, which showed a response rate of $29 \%$ in tumors that were PD-L1 negative. ${ }^{82}$ This study randomizes PD-L1-negative patients to one of the following three arms: combined PD-L1 (durvalumab)/ CTLA-4 (tremelimumab) inhibitor, monotherapy with PD-L1 inhibitor, and monotherapy with CTLA-4 inhibitor. Another ongoing randomized study addressing this combination is EAGLE (NCT02369874), which is a confirmatory trial in both PD-L1-positive and -negative patients who have progressed on platinum therapy. This study, similar to the frontline randomized study, is a three-arm trial randomizing patients to the combination of PD-L1/CTLA-4 inhibitor vs PD-L1 inhibitor alone vs standard of care chemotherapy.

KESTREL (NCT02551159) is a three-arm, Phase III randomized open-label study in first-line treatment of recurrent/ metastatic HNSCC comparing durvalumab (PD-L1 inhibitor) with tremelimumab (CTLA-4 inhibitor) combination (arm 1) to standard of care treatment with chemotherapy (arm 2) to single-agent durvalumab (arm 3). This study also includes both PD-L1 positive and negative patients. Similarly, CheckMate-651 (NCT02741570) is evaluating nivolumab and ipilimumab in the first-line setting and CheckMate-714 (NCT02823574) is randomizing the same population to receive either nivolumab and ipilimumab combination or nivolumab/placebo. However, PD-1 inhibitors and CTLA-4 inhibitors have previously demonstrated concerns for added toxicity as seen in the melanoma patients with a grade 3 or 4 AE rate of $55 \%{ }^{83}$ compared to $16 \%$ for single-agent PD-1 inhibitors and $27 \%$ for CTLA-4 inhibitors. ${ }^{83}$ This would need to be closely monitored in HNSCC patients as they often have significant smoking and age-related comorbidities.

PD-1 inhibitors are also being combined with other checkpoint receptors including LAG3 and killer-cell immunoglobulin receptors (KIRs). LAG3 enhances function of Tregs and prevents $\mathrm{CD} 8^{+} \mathrm{T}$-cell activation and is expressed along with PD-1 on exhausted T cells. ${ }^{84}$ KIRs are expressed by NK cells, interact with $\mathrm{MHC} 1$, and reduce $\mathrm{NK}$-cell activity. ${ }^{85}$ Phase I trials are ongoing with both anti-KIR (NCT01714739) and anti-LAG3 (NCT01968109) antibodies in combination with PD-1 inhibitors in solid tumors including HNSCC.
CTLA-4 inhibitors are being combined with other immune-modulating drugs. Enoblituzumab, a humanized monoclonal antibody directed against CD276 (part of B7-H3 family of negative checkpoint receptors on APCs), is currently being combined with ipilimumab in HNSCC and other solid tumors expressing B7-H3 (NCT01860430 and NCT01935921). Ipilimumab is also being combined with radiation and cetuximab in HPV-positive locally advanced disease (NCT01935921).

\section{PD-I inhibitors in combination with traditional oncological therapies}

Chemotherapy is effective at cancer cell death, but durable responses are not often seen with historical cytotoxic therapy. The advent of immunotherapy has led to a better understanding of the relationship between the immune system and cell death as a result of chemotherapy and radiation therapy. Cytotoxic or radiation regimens that result in cancer cell death can ignite immune responses through a process called immunogenic cell death. This process is associated with calreticulin expression on dying cells, increase in TAAs that are presented to APCs, and release of high mobility group box 1 (HMGB1). ${ }^{86}$ In addition, surviving tumor cells undergo changes that make these cells more amenable to immunemediated cell death by reducing expression of survival genes and alterations in the antigen-processing machinery. ${ }^{86}$ Radiation therapy has also been associated with abscopal effects, namely increasing T-cell infiltration into the TME, increased TAA presentation, and secretion of cytokines. These changes provide a biological rationale for combining traditional chemotherapy and immunotherapy to further augment the immune-mediated cell death. A number of clinical trials are ongoing combining both chemotherapy and radiation with pembrolizumab and other PD-1 inhibitors (Table 1).

\section{PD-I inhibitors in combination with DNA repair and transcription-modulating agents PARP inhibitors}

PARP is involved in several forms of DNA repair (including single strand, double strand, and base excision repair) and is considered as an important survival pathway for cancer cells. ${ }^{87,88}$ This has led to the development of a number of PARP inhibitors that are currently approved in BRCA-positive ovarian cancer and is also being evaluated in a number of malignancies with DNA repair defects.

Although germline mutations in DNA repair pathways are not commonly associated with head and neck cancers, 
Table I Pembrolizumab: active Phase II and Phase III clinical trials

\begin{tabular}{|c|c|c|c|}
\hline Clinical trial & Phase & Patient population & Study design \\
\hline NCT0235803। KEYNOTE-048 & III & Recurrent or metastatic HNSCC & $\begin{array}{l}\text { Pembrolizumab alone or with platinum }+5 \text {-FU, or } \\
\text { platinum }+5 \text {-FU }+ \text { cetuximab }\end{array}$ \\
\hline NCT02252042 KEYNOTE-040 & III & Recurrent or metastatic HNSCC & Pembrolizumab vs cetuximab, docetaxel, or methotrexate \\
\hline NCT02759575 & $1 / I I$ & Locally advanced laryngeal cancer & Pembrolizumab + cisplatin and + radiation \\
\hline NCT0264I093 & II & Resectable HNSCC & Pembrolizumab \pm cisplatin and radiation following surgery \\
\hline NCT02769520 & II & Relapsed, locally advanced HNSCC & Pembrolizumab after salvage surgical resection \\
\hline NCT02289209 & II & $\begin{array}{l}\text { Loco-regional inoperable recurrence or } \\
\text { the second primary HNSCC }\end{array}$ & Pembrolizumab + re-irradiation \\
\hline NCT02777385 & II & $\begin{array}{l}\text { Intermediate or high risk, previously } \\
\text { untreated, locally advanced HNSCC }\end{array}$ & $\begin{array}{l}\text { Pembrolizumab }+ \text { concurrent cisplatin and radiation or } \\
\text { pembrolizumab followed by cisplatin and radiation }\end{array}$ \\
\hline NCT0289220I & II & Primary HNSCC & Pembrolizumab given if residual disease seen after radiation \\
\hline NCT025385I0 & $\mathrm{I} / \mathrm{II}$ & $\begin{array}{l}\text { Metastatic/unresectable HNSCC and } \\
\text { salivary gland tumors }\end{array}$ & Pembrolizumab combined with vorinostat \\
\hline NCT023 I877। & 1 & Metastatic HNSCC and other solid tumors & $\begin{array}{l}\text { Pembrolizumab with radiation to assess } \\
\text { immunostimulatory action }\end{array}$ \\
\hline NCT02296684 & II & Locally advanced operable HNSCC & $\begin{array}{l}\text { Pembrolizumab + cisplatin + radiation after surgical } \\
\text { resection }\end{array}$ \\
\hline NCT02454I79 & II & Advanced HNSCC & Combining ACP-196 and pembrolizumab \\
\hline NCT02626000 & 1 & Recurrent or metastatic HNSCC & Talimogene laherparepvec with pembrolizumab \\
\hline NCT02609503 & II & Locally advanced HNSCC & $\begin{array}{l}\text { Pembrolizumab and radiation in those who are not eligible } \\
\text { for cisplatin }\end{array}$ \\
\hline NCT02586207 & 1 & Locally advanced HNSCC & Pembrolizumab and chemoradiation \\
\hline NCT024752। 3 & 1 & Metastatic HNSCC and other solid tumors & $\begin{array}{l}\text { Combining pembrolizumab with MGA27I in patients } \\
\text { expressing B7-H3 }\end{array}$ \\
\hline NCT026II 960 & II & Recurrent nasopharyngeal cancer & Pembrolizumab vs standard platinum therapy \\
\hline NCT02 178722 & $\mathrm{I} / \mathrm{II}$ & Solid tumors including HNSCC & Pembrolizumab in combination with INCB024360 \\
\hline NCT02452424 & $\mathrm{I} / \mathrm{II}$ & Solid tumors including HNSCC & $\begin{array}{l}\text { Double immune-suppression blockade in combination with } \\
\text { CSFIR inhibitor }\end{array}$ \\
\hline
\end{tabular}

Abbreviations: 5-FU, 5- Fluorouracil; HNSCC, head and neck squamous cell cancer.

somatic mutations and alterations in pathways related to DNA repair are commonly seen in different subsites of HNSCC. ${ }^{7,89}$ Preclinical data suggest increased response to PARP inhibition in head and neck cancers with a defect in DNA repair pathways ${ }^{90}$ and activity in squamous cell cancer of the lung after platinum exposure and resistance. ${ }^{91}$ Furthermore, some studies suggest that HPV-positive tumors appear to have an increased sensitivity to PARP inhibitors. ${ }^{92}$ PARP is also involved in inflammatory responses, by regulating the immunosuppressive effects of Tregs. ${ }^{93}$ As a result of these properties, the combination of PARP inhibitors and PD-1 inhibitors remains an area of interest in malignancies that harbor DNA repair defects such as prostate cancer (NCT02861573) but would also provide a biological rationale in HNSCC.

\section{PD-I inhibitors with histone de-acetylase (HDAC) inhibitors}

HDAC removes the acetyl groups, causing hypoacetylation of histones, which in turn results in a condensed chromatin structure and suppression of gene transcription. HDAC inhibitors cause accumulation of acetyl groups on the histone lysine, allowing open chromatin structure and transcriptional activation and gene expression. HDAC inhibitors can cause tumor cell growth arrest, apoptosis, inhibit growth of tumors, and are widely used in a variety of malignancies.

Epigenetic modulation by HDAC inhibitors has also been identified to have potential immunomodulatory activity. Studies have shown that HDAC inhibitors increase the expression of $\mathrm{MHC}^{94}$ and costimulatory molecules ${ }^{95}$ as well as augment INF- $\gamma$ production ${ }^{96}$ and promote CTL-mediated tumor death. ${ }^{94}$ Preclinical tumor models have also shown that combining HDAC inhibitors with immune-modulating drugs inhibited Treg activity, improved APCs functionality, and potentiated proliferation and survival of CTLs resulting in enhanced antitumor immune response. ${ }^{97,98}$ In addition, leukemia cell lines treated with epigenetic agents led to upregulation of PD-L1, PD-L2, and CTLA-4 expressions and may be a reason for developing resistance to these drugs. ${ }^{99}$ These unique properties of HDAC inhibitors have led to clinical trials involving vorinostat and pembrolizumab in lung cancer, renal cell cancer, as well as HNSCC and salivary gland tumors (NCT02538510). 


\section{Immunotherapy future in HNSCC}

It is clear that pembrolizumab, along with other immunemodulating drugs, has changed the treatment landscape in HNSCC as shown by multiple clinical trials demonstrating effectiveness in comparison to conventional cytotoxic chemotherapy. ${ }^{57,59,60}$ The ability of these drugs to induce durable responses with manageable toxicities in this patient group represents an exciting development in the field of head and neck oncology.

There are a number of issues that need to be addressed, namely how benefits of these drugs should be measured. Response rate has been a valid historical end point in recurrent and metastatic trials; however, it may not be the best end point for immune-modulating studies. In regard to pembrolizumab, improved response led to approval of the drug $\mathrm{g}^{59,60,66}$ and, while response rates were much better than the 3\%-13\% historical rates with single-agent chemotherapy, response rate with single-agent PD-1 inhibitor remains relatively low $(10 \%-20 \%)^{57,59,60}$ Although not all patients respond to these drugs (unlike conventional cytotoxic therapy where duration of response is generally short lived), these immunemodulating drugs can produce long durable responses in many responders and, therefore, skew PFS measurements. ${ }^{57}$ For example, in the KEYNOTE-012 study, the median duration of response was 53 weeks $^{59}$ and, in the expansion cohort study, it was not reached and a number of patients still had ongoing responses at the completion of each of the trials. ${ }^{60}$ If response is of interest in design of future trials, it is important to consider durability of response in addition to response rate as a primary end point.

Nivolumab is the only checkpoint inhibitor to this date that has randomized data reported demonstrating OS benefit in HNSCC. Although median survival in this study was relatively short (median 7.5 months vs 5.1 months in the chemotherapy arm), it is important to remember that the patient population was a high-risk group of pretreated patients who progressed shortly after platinum. More impressively, review of Kaplan-Meier curves reveals a suggestion of "tail" in the nivolumab curve and a small group of long-term survivors. The 1-year estimate of survival in this study was clearly in favor of nivolumab $(36.0 \%$ vs $16.6 \%$ in the chemotherapy arm), was nearly doubled, and was similar to survivals that were historically achieved in the first-line untreated patients. Perhaps arguing percentage of long-term survivors is a more valid end point than median survival time.

Interestingly, the survival advantage in this study was achieved in light of no statistically significant PFS benefit (median 2.0 vs 2.3 months for nivolumab vs chemotherapy) ${ }^{57}$ suggesting that some patients still benefit from nivolumab, despite lack of radiographic response. Therefore it remains imperative that OS should remain as the gold standard to measure effectiveness of these drugs regardless of the impressive response rates.

In light of improving clinical efficacy, the need to identify a predictive biomarker remains a significant challenge. Currently, PD-L1 expression on tumor cells is the only marker that has been commonly reported. However, it is well known that a small group of patients who have low PD-L1 expression can still respond to pembrolizumab and, while reasons for this remain unclear, an emerging concept of dynamic PD-L1 expression in the evolution of a malignancy ${ }^{86}$ may in some part explain this phenomenon. This can be seen in the CheckMate-141 study where patients with $<1 \%$ PD-L1 expression still had a $12 \%$ $\mathrm{ORR}^{57}$ when treated with nivolumab and in the KEYNOTE-012 study where there was an ORR of $4 \%$ in patients with PD-L1 expression $<1 \% .{ }^{60}$ In addition, an inflammatory gene signature that includes expression of INF- $\gamma$ has shown impressive rates of predicting nonresponders treated with pembrolizumab in both $\mathrm{HNSCC}^{59}$ and melanoma patients, ${ }^{100}$ but larger datasets are needed to further validate this signature.

Another important development is identifying rational combinations that can improve clinical benefit especially in those who are low PD-L1 expressers. A number of new checkpoint drugs are in development or are in early trials with pembrolizumab, and the results of these trials are eagerly anticipated. Furthermore, a number of clinical trials involving chemotherapy and radiation with pembrolizumab are currently ongoing in patients with early-stage HNSCC with the rationale of increasing neoantigen presentation and increasing PD-L1 expression and, therefore, improving outcomes.

\section{Conclusion}

The advent of PD-1 immunotherapy has led to new and exciting times for patients with recurrent/metastatic HNSCC with significantly improved outcomes compared to chemotherapy or epidermal growth factor inhibitor treatment. However, response rates remain low in the $10 \%-20 \%$ range across various checkpoint inhibitors and a better understanding of resistance mechanisms is needed to improve responses. PD-L1 expression is an extensively studied biomarker that suggests some correlation with response as high expressers demonstrate better responses compared to patients with low PD-L1 expression. However, there are reports of patients who are high PD-L1 expressers who do not respond and, conversely, patients with low PD-L1 expression can have robust responses to anti-PD-1 therapy, suggesting that 
PD-L1 expression at this time should not be used for making treatment decisions in HNSCC, but instead as a biomarker that needs to be further validated through studies and as a guidance for the design of future trials. It is also not clear if a radiographic response is required to drive long-term survival benefit from these treatments and whether maximizing response will translate into durable survival. As a result, more accurate biomarkers are needed that can better predict outcome, increase sensitivity of tumor cells to checkpoint inhibitors, and assist in design of a future wave of studies. Currently, there are a number of trials underway using novel combinations with chemotherapy, radiation and other checkpoint inhibitors or DNA-modulating agents to help improve responses and outcomes in HNSCC.

\section{Disclosure}

The authors report no conflicts of interest, financial, or otherwise with respect to this work.

\section{References}

1. Parkin DM, Bray F, Ferlay J, Pisani P. Global cancer statistics, 2002. CA Cancer J Clin. 2005;55(2):74-108.

2. Parkin DM, Muir CS. Cancer incidence in five continents. Comparability and quality of data. IARC Sci Publ. 1992;120:45-173.

3. Brockstein B, Haraf DJ, Rademaker AW, et al. Patterns of failure, prognostic factors and survival in locoregionally advanced head and neck cancer treated with concomitant chemoradiotherapy: a 9-year, 337-patient, multi-institutional experience. Ann Oncol. 2004;15(8): 1179-1186.

4. Vermorken JB, Mesia R, Rivera F, et al. Platinum-based chemotherapy plus cetuximab in head and neck cancer. $N$ Engl J Med. 2008; 359(11):1116-1127.

5. Vermorken JB, Stohlmacher-Williams J, Davidenko I, et al. Cisplatin and fluorouracil with or without panitumumab in patients with recurrent or metastatic squamous-cell carcinoma of the head and neck (SPECTRUM): an open-label phase 3 randomised trial. Lancet Oncol. 2013;14(8):697-710.

6. Saloura V, Cohen EE, Licitra L, et al. An open-label single-arm, phase II trial of zalutumumab, a human monoclonal anti-EGFR antibody, in patients with platinum-refractory squamous cell carcinoma of the head and neck. Cancer Chemother Pharmacol. 2014;73(6):1227-1239.

7. Romick-Rosendale LE, Lui VW, Grandis JR, Wells SI. The Fanconi anemia pathway: repairing the link between DNA damage and squamous cell carcinoma. Mutat Res. 2013;743-744:78-88.

8. Chikamatsu K, Albers A, Stanson J, et al. P53(110-124)-specific human CD4+ T-helper cells enhance in vitro generation and antitumor function of tumor-reactive CD8+ T cells. Cancer Res. 2003;63(13): 3675-3681.

9. Gillison ML, D'Souza G, Westra W, et al. Distinct risk factor profiles for human papillomavirus type 16-positive and human papillomavirus type 16-negative head and neck cancers. J Natl Cancer Inst. 2008; 100(6):407-420.

10. Sckisel GD, Bouchlaka MN, Monjazeb AM, et al. Out-of-sequence signal 3 paralyzes primary CD4(+) T-cell-dependent immunity. Immunity. 2015;43(2):240-250.

11. Moy JD, Moskovitz JM, Ferris RL. Biological mechanisms of immune escape and implications for immunotherapy in head and neck squamous cell carcinoma. Eur J Cancer. 2017;76:152-166.

12. Burnet FM. The concept of immunological surveillance. Prog Exp Tumor Res. 1970;13:1-27.
13. Bhatia $\mathrm{S}$, Louie $\mathrm{AD}$, Bhatia $\mathrm{R}$, et al. Solid cancers after bone marrow transplantation. J Clin Oncol. 2001;19(2):464-471.

14. Haigentz M Jr. Aerodigestive cancers in HIV infection. Curr Opin Oncol. 2005;17(5):474-478.

15. Ljunggren $\mathrm{HG}$, Karre K. In search of the 'missing self': MHC molecules and NK cell recognition. Immunol Today. 1990;11(7):237-244.

16. Lopez-Albaitero A, Nayak JV, Ogino T, et al. Role of antigen-processing machinery in the in vitro resistance of squamous cell carcinoma of the head and neck cells to recognition by CTL. J Immunol. 2006;176(6): 3402-3409.

17. Ferris RL, Whiteside TL, Ferrone S. Immune escape associated with functional defects in antigen-processing machinery in head and neck cancer. Clin Cancer Res. 2006;12(13):3890-3895.

18. Concha-Benavente F, Srivastava R, Ferrone S, Ferris RL. Immunological and clinical significance of HLA class I antigen processing machinery component defects in malignant cells. Oral Oncol. 2016; 58:52-58.

19. Meissner M, Reichert TE, Kunkel M, et al. Defects in the human leukocyte antigen class I antigen processing machinery in head and neck squamous cell carcinoma: association with clinical outcome. Clin Cancer Res. 2005;11(7):2552-2560.

20. Mimura K, Shiraishi K, Mueller A, et al. The MAPK pathway is a predominant regulator of HLA-A expression in esophageal and gastric cancer. J Immunol. 2013;191(12):6261-6272.

21. Lechner MG, Megiel C, Russell SM, et al. Functional characterization of human $\mathrm{Cd} 33+$ and $\mathrm{Cd} 11 \mathrm{~b}+$ myeloid-derived suppressor cell subsets induced from peripheral blood mononuclear cells co-cultured with a diverse set of human tumor cell lines. J Transl Med. 2011;9:90.

22. Jie HB, Gildener-Leapman N, Li J, et al. Intratumoral regulatory T cells upregulate immunosuppressive molecules in head and neck cancer patients. Br J Cancer. 2013;109(10):2629-2635.

23. Chikamatsu K, Sakakura K, Toyoda M, Takahashi K, Yamamoto T, Masuyama K. Immunosuppressive activity of CD14+ HLA-DR-cells in squamous cell carcinoma of the head and neck. Cancer Sci. 2012; 103(6):976-983.

24. Vasquez-Dunddel D, Pan F, Zeng Q, et al. STAT3 regulates arginase-I in myeloid-derived suppressor cells from cancer patients. J Clin Invest. 2013;123(4):1580-1589.

25. Kuss I, Hathaway B, Ferris RL, Gooding W, Whiteside TL. Decreased absolute counts of T lymphocyte subsets and their relation to disease in squamous cell carcinoma of the head and neck. Clin Cancer Res. 2004;10(11):3755-3762.

26. Dasgupta S, Bhattacharya-Chatterjee M, O’Malley BW Jr, Chatterjee SK. Inhibition of NK cell activity through TGF-beta 1 by down-regulation of NKG2D in a murine model of head and neck cancer. J Immunol. 2005;175(8):5541-5550.

27. Muenst S, Laubli H, Soysal SD, Zippelius A, Tzankov A, Hoeller S. The immune system and cancer evasion strategies: therapeutic concepts. J Intern Med. 2016;279(6):541-562.

28. Fuereder T. Immunotherapy for head and neck squamous cell carcinoma. Memo. 2016;9:66-69.

29. Whiteside TL. Immune cells in the tumor microenvironment. Mechanisms responsible for functional and signaling defects. Adv Exp Med Biol. 1998;451:167-171.

30. Ferris RL. Immunology and immunotherapy of head and neck cancer. J Clin Oncol. 2015;33(29):3293-3304.

31. Baruah P, Lee M, Odutoye T, et al. Decreased levels of alternative costimulatory receptors OX40 and 4-1BB characterise T cells from head and neck cancer patients. Immunobiology. 2012;217(7):669-675.

32. Schwartz RH. Costimulation of T lymphocytes: the role of CD28, CTLA-4, and B7/BB1 in interleukin-2 production and immunotherapy. Cell. 1992;71(7):1065-1068.

33. Pentcheva-Hoang T, Simpson TR, Montalvo-Ortiz W, Allison JP. Cytotoxic T lymphocyte antigen-4 blockade enhances antitumor immunity by stimulating melanoma-specific T-cell motility. Cancer Immunol Res. 2014;2(10):970-980. 
34. Chan DV, Gibson HM, Aufiero BM, et al. Differential CTLA-4 expression in human CD4+ versus CD8+ T cells is associated with increased NFAT1 and inhibition of CD4+ proliferation. Genes Immun. 2014;15(1):25-32.

35. Takahashi $T$, Tagami $T$, Yamazaki $S$, et al. Immunologic self-tolerance maintained by $\mathrm{CD} 25(+) \mathrm{CD} 4(+)$ regulatory $\mathrm{T}$ cells constitutively expressing cytotoxic T lymphocyte-associated antigen 4. J Exp Med. 2000;192(2):303-310.

36. Wing K, Onishi Y, Prieto-Martin P, et al. CTLA-4 control over Foxp3+ regulatory T cell function. Science. 2008;322(5899):271-275.

37. Qureshi OS, Zheng Y, Nakamura K, et al. Trans-endocytosis of CD 80 and CD86: a molecular basis for the cell-extrinsic function of CTLA-4. Science. 2011;332(6029):600-603.

38. Oderup C, Cederbom L, Makowska A, Cilio CM, Ivars F. Cytotoxic T lymphocyte antigen-4-dependent down-modulation of costimulatory molecules on dendritic cells in CD4+CD25+ regulatory T-cell-mediated suppression. Immunology. 2006;118(2):240-249.

39. Leach DR, Krummel MF, Allison JP. Enhancement of antitumor immunity by CTLA-4 blockade. Science. 1996;271(5256):1734-1736.

40. Hodi FS, O'Day SJ, McDermott DF, et al. Improved survival with ipilimumab in patients with metastatic melanoma. $N$ Engl J Med. 2010; 363(8):711-723.

41. ClinicalTrials.gov. 2017. Accessed 27 April, 2017.

42. Keir ME, Butte MJ, Freeman GJ, Sharpe AH. PD-1 and its ligands in tolerance and immunity. Апnи Rev Immunol. 2008;26:677-704.

43. Keir ME, Liang SC, Guleria I, et al. Tissue expression of PD-L1 mediates peripheral T cell tolerance. $J$ Exp Med. 2006;203(4):883-895.

44. Eppihimer MJ, Gunn J, Freeman GJ, et al. Expression and regulation of the PD-L1 immunoinhibitory molecule on microvascular endothelial cells. Microcirculation. 2002;9(2):133-145.

45. Latchman Y, Wood CR, Chernova T, et al. PD-L2 is a second ligand for PD-1 and inhibits T cell activation. Nat Immunol. 2001;2(3): 261-268.

46. Butte MJ, Keir ME, Phamduy TB, Sharpe AH, Freeman GJ. Programmed death-1 ligand 1 interacts specifically with the B7-1 costimulatory molecule to inhibit T cell responses. Immunity. 2007;27(1):111-122.

47. Iwai $Y$, Ishida $M$, Tanaka $Y$, Okazaki $T$, Honjo $T$, Minato N. Involvement of PD-L1 on tumor cells in the escape from host immune system and tumor immunotherapy by PD-L1 blockade. Proc Natl Acad Sci US A. 2002;99(19):12293-12297.

48. Strome SE, Dong H, Tamura H, et al. B7-H1 blockade augments adoptive T-cell immunotherapy for squamous cell carcinoma. Cancer Res. 2003;63(19):6501-6505.

49. Chen J, Feng Y, Lu L, et al. Interferon-gamma-induced PD-L1 surface expression on human oral squamous carcinoma via PKD2 signal pathway. Immunobiology. 2012;217(4):385-393.

50. Concha-Benavente F, Srivastava RM, Trivedi S, et al. Identification of the cell-intrinsic and -extrinsic pathways downstream of EGFR and IFNgamma that induce PD-L1 expression in head and neck cancer. Cancer Res. 2016;76(5):1031-1043.

51. Parsa AT, Waldron JS, Panner A, et al. Loss of tumor suppressor PTEN function increases B7-H1 expression and immunoresistance in glioma. Nat Med. 2007;13(1):84-88.

52. Ghebeh H, Mohammed S, Al-Omair A, et al. The B7-H1 (PD-L1) T lymphocyte-inhibitory molecule is expressed in breast cancer patients with infiltrating ductal carcinoma: correlation with important high-risk prognostic factors. Neoplasia. 2006;8(3):190-198.

53. Lalami Y, Awada A. Innovative perspectives of immunotherapy in head and neck cancer. From relevant scientific rationale to effective clinical practice. Cancer Treat Rev. 2016;43:113-123.

54. Thompson RH, Gillett MD, Cheville JC, et al. Costimulatory molecule B7-H1 in primary and metastatic clear cell renal cell carcinoma. Cancer. 2005;104(10):2084-2091

55. Thompson RH, Gillett MD, Cheville JC, et al. Costimulatory B7-H1 in renal cell carcinoma patients: indicator of tumor aggressiveness and potential therapeutic target. Proc Natl Acad Sci U S A. 2004;101(49): 17174-17179.
56. Tsushima F, Tanaka K, Otsuki N, et al. Predominant expression of B7-H1 and its immunoregulatory roles in oral squamous cell carcinoma. Oral Oncol. 2006;42(3):268-274.

57. Ferris RL, Blumenschein G Jr, Fayette J, et al. Nivolumab for recurrent squamous-cell carcinoma of the head and neck. N Engl J Med. 2016; 375(19):1856-1867.

58. Parikh F, Duluc D, Imai N, et al. Chemoradiotherapy-induced upregulation of PD-1 antagonizes immunity to HPV-related oropharyngeal cancer. Cancer Res. 2014;74(24):7205-7216.

59. Seiwert TY, Burtness B, Mehra R, et al. Safety and clinical activity of pembrolizumab for treatment of recurrent or metastatic squamous cell carcinoma of the head and neck (KEYNOTE-012): an open-label, multicentre, phase 1b trial. Lancet Oncol. 2016;17(7):956-965.

60. Chow LQ, Haddad R, Gupta S, et al. Antitumor activity of pembrolizumab in biomarker-unselected patients with recurrent and/or metastatic head and neck squamous cell carcinoma: results from the phase $\mathrm{Ib}$ KEYNOTE-012 expansion cohort. J Clin Oncol. 2016.

61. Hamid O, Robert C, Daud A, et al. Safety and tumor responses with lambrolizumab (anti-PD-1) in melanoma. $N$ Engl J Med. 2013; 369(2):134-144.

62. Robert C, Schachter J, Long GV, et al. Pembrolizumab versus ipilimumab in advanced melanoma. N Engl J Med. 2015;372(26):2521-2532.

63. Garon EB, Rizvi NA, Hui R, et al. Pembrolizumab for the treatment of non-small-cell lung cancer. N Engl J Med. 2015;372(21):2018-2028.

64. Herbst RS, Baas P, Kim DW, et al. Pembrolizumab versus docetaxel for previously treated, PD-L1-positive, advanced non-small-cell lung cancer (KEYNOTE-010): a randomised controlled trial. Lancet. 2016; 387(10027):1540-1550.

65. Reck M, Rodriguez-Abreu D, Robinson AG, et al. Pembrolizumab versus chemotherapy for PD-L1-positive non-small-cell lung cancer. N Engl J Med. 2016;375(19):1823-1833.

66. Bauml J, Seiwert TY, Pfister DG, et al. Pembrolizumab for platinumand cetuximab-refractory head and neck cancer: results from a singlearm, phase II study. J Clin Oncol. 2017;35(14):1542-1549.

67. Au E, Ang PT. A phase II trial of 5-fluorouracil and cisplatinum in recurrent or metastatic nasopharyngeal carcinoma. Ann Oncol. 1994; $5(1): 87-89$.

68. Rusthoven CG, Lanning RM, Jones BL, et al. Metastatic nasopharyngeal carcinoma: patterns of care and survival for patients receiving chemotherapy with and without local radiotherapy. Radiother Oncol. 2017; 124(1):139-146.

69. Hsu Cea. Antitumor Activity and Safety of Pembrolizumab in Patients with PD-L1-Positive Nasopharyngeal Carcinoma: Interim Results from a Phase 1b Study ESMO; 12/09/2015. Vienna, Austria: 2015.

70. Cho YA, Yoon HJ, Lee JI, Hong SP, Hong SD. Relationship between the expressions of PD-L1 and tumor-infiltrating lymphocytes in oral squamous cell carcinoma. Oral Oncol. 2011;47(12):1148-1153.

71. Lin YM, Sung WW, Hsieh MJ, et al. High PD-L1 expression correlates with metastasis and poor prognosis in oral squamous cell carcinoma. PLoS One. 2015;10(11): 0142656.

72. Chow L, Mehra R, Haddad R, et al. Antitumor activity of the antiPD-1 antibody pembrolizumab in subgroups of patients with recurrent/ metastatic head and neck squamous cell carcinoma (R/M HNSCC): exploratory analyses from KEYNOTE-012. European Cancer Congress. Vienna: 2015.

73. Hanna GJ, Liu H, Jones RE, et al. Defining an inflamed tumor immunophenotype in recurrent, metastatic squamous cell carcinoma of the head and neck. Oral Oncol. 2017;67:61-69.

74. Tumeh PC, Harview CL, Yearley JH, et al. PD-1 blockade induces responses by inhibiting adaptive immune resistance. Nature. 2014; 515(7528):568-571.

75. Ang KK, Harris J, Wheeler R, et al. Human papillomavirus and survival of patients with oropharyngeal cancer. $N$ Engl $J$ Med. 2010;363(1):24-35.

76. Keck MK, Zuo Z, Khattri A, et al. Integrative analysis of head and neck cancer identifies two biologically distinct HPV and three non-HPV subtypes. Clin Cancer Res. 2015;21(4):870-881. 
77. Lyford-Pike S, Peng S, Young GD, et al. Evidence for a role of the PD-1:PD-L1 pathway in immune resistance of HPV-associated head and neck squamous cell carcinoma. Cancer Res. 2013;73(6):1733-1741.

78. Weber JS, O'Day S, Urba W, et al. Phase I/II study of ipilimumab for patients with metastatic melanoma. J Clin Oncol. 2008;26(36): 5950-5956.

79. Wolchok JD, Hoos A, O'Day S, et al. Guidelines for the evaluation of immune therapy activity in solid tumors: immune-related response criteria. Clin Cancer Res. 2009;15(23):7412-7420.

80. Hashemi-Sadraei N, Sikora AG, Brizel DM. Immunotherapy and checkpoint inhibitors in recurrent and metastatic head and neck cancer. Am Soc Clin Oncol Educ Book. 2016;35:e277-e282.

81. Curran MA, Montalvo W, Yagita H, Allison JP. PD-1 and CTLA-4 combination blockade expands infiltrating $\mathrm{T}$ cells and reduces regulatory T and myeloid cells within B16 melanoma tumors. Proc Natl Acad Sci US A. 2010;107(9):4275-4280.

82. Antonia S, Goldberg SB, Balmanoukian A, et al. Safety and antitumour activity of durvalumab plus tremelimumab in non-small cell lung cancer: a multicentre, phase 1b study. Lancet Oncol. 2016;17(3):299-308.

83. Larkin J, Chiarion-Sileni V, Gonzalez R, et al. Combined nivolumab and ipilimumab or monotherapy in untreated melanoma. N Engl J Med. 2015;373(1):23-34.

84. Grosso JF, Goldberg MV, Getnet D, et al. Functionally distinct LAG-3 and PD-1 subsets on activated and chronically stimulated CD8 T cells. J Immunol. 2009;182(11):6659-6669.

85. Thielens A, Vivier E, Romagne F. NK cell MHC class I specific receptors (KIR): from biology to clinical intervention. Curr Opin Immunol. 2012;24(2):239-245

86. Bauman JE, Cohen E, Ferris RL, et al. Immunotherapy of head and neck cancer: emerging clinical trials from a national cancer institute head and neck cancer steering committee planning meeting. Cancer. 2017; 123(7):1259-1271.

87. Farmer H, McCabe N, Lord CJ, et al. Targeting the DNA repair defect in BRCA mutant cells as a therapeutic strategy. Nature. 2005; 434(7035):917-921.

88. Lee JM, Ledermann JA, Kohn EC. PARP Inhibitors for BRCA1/2 mutation-associated and BRCA-like malignancies. Ann Oncol. 2014; 25(1):32-40.

89. Hashemi-Sadraei N, Kakajan K, Egloff A, et al. Prevalence and Outcome of Mutations (mut) in the Fanconi Anemia (FA) DNA Repair Pathway Among Head and Neck Cancer (H\&N Ca) Patients (pts). Chicago: ASCO; 2014.
90. Lombardi AJ, Hoskins EE, Foglesong GD, et al. Acquisition of relative interstrand crosslinker resistance and PARP inhibitor sensitivity in fanconi anemia head and neck cancers. Clin Cancer Res. 2015;21(8): 1962-1972.

91. Michels J, Vitale I, Galluzzi L, et al. Cisplatin resistance associated with PARP hyperactivation. Cancer Res. 2013;73(7):2271-2280.

92. Weaver AN, Cooper TS, Rodriguez M, et al. DNA double strand break repair defect and sensitivity to poly ADP-ribose polymerase (PARP) inhibition in human papillomavirus 16-positive head and neck squamous cell carcinoma. Oncotarget. 2015;6(29):26995-27007.

93. Zhang P, Maruyama T, Konkel JE, et al. PARP-1 controls immunosuppressive function of regulatory T cells by destabilizing Foxp3. PLoS One. 2013;8(8):e71590.

94. Manning J, Indrova M, Lubyova B, et al. Induction of MHC class I molecule cell surface expression and epigenetic activation of antigen-processing machinery components in a murine model for human papilloma virus 16-associated tumours. Immunology. 2008; 123(2):218-227.

95. Maeda T, Towatari M, Kosugi H, Saito H. Up-regulation of costimulatory/adhesion molecules by histone deacetylase inhibitors in acute myeloid leukemia cells. Blood. 2000;96(12):3847-3856.

96. Khan AN, Gregorie CJ, Tomasi TB. Histone deacetylase inhibitors induce TAP, LMP, Tapasin genes and MHC class I antigen presentation by melanoma cells. Cancer Immunol Immunother. 2008;57(5): 647-654.

97. Christiansen AJ, West A, Banks KM, et al. Eradication of solid tumors using histone deacetylase inhibitors combined with immunestimulating antibodies. Proc Natl Acad Sci U S A. 2011;108(10): 4141-4146.

98. Shen L, Ciesielski M, Ramakrishnan S, et al. Class I histone deacetylase inhibitor entinostat suppresses regulatory $\mathrm{T}$ cells and enhances immunotherapies in renal and prostate cancer models. PLoS One. 2012;7(1):e30815

99. Yang H, Bueso-Ramos C, DiNardo C, et al. Expression of PD-L1, PD-L2, PD-1 and CTLA4 in myelodysplastic syndromes is enhanced by treatment with hypomethylating agents. Leukemia. 2014;28(6): 1280-1288.

100. Ribas A, Caroline Robert F, Hodi S, et al. Association of Response to Programmed Death Receptor 1 (PD-1) Blockade with Pembrolizumab (MK-3475) with an Interferon-Inflammatory Immune Gene Signature. Chicago: ASCO; 2015.

\section{Publish your work in this journal}

Drug Design, Development and Therapy is an international, peerreviewed open-access journal that spans the spectrum of drug design and development through to clinical applications. Clinical outcomes, patient safety, and programs for the development and effective, safe, and sustained use of medicines are the features of the journal, which

\section{Dovepress}

has also been accepted for indexing on PubMed Central. The manuscript management system is completely online and includes a very quick and fair peer-review system, which is all easy to use. Visit http://www.dovepress.com/testimonials.php to read real quotes from published authors. 